\title{
De la planificación estratégica al marketing urbano: hacia la ciudad inmaterial
}

Andrés Precedo Ledo. Catedrático de Geografía Humana. Departamento de Geografía, Universidad de Santiago de Compostela.

José Javier Orosa González. Profesor de Comercialización e Investigación de Mercados. Departamento de Análisis Económico y Administración de Empresas, Universidad da Coruña.

Alberto Míguez Iglesias. Departamento de Geografia, Universidad de Santiago de Compostela.

RESUMEN | El presente artículo hace una revisión de cómo han realizado distintos tipos de ciudades su posicionamiento en el sistema global mediante el estudio de casos significativos.

En la parte empírica, se realiza un análisis comparativo entre las actuaciones urbanas realizadas por 37 ciudades en dos periodos de tiempo diferentes: entre 1980 y 1995 , que corresponde a la etapa de transición industrial, y entre 1995 y 2005, que responde a la etapa postindustrial.

Se concluye que aquellas actuaciones vinculadas a la calidad de vida urbana y al marketing urbano han ido adquiriendo mayor relevancia que otras más vinculadas a la planificación estratégica de carácter económico. Asimismo, las actuaciones de marcado carácter material dejan paso a otras más relacionadas con aspectos inmateriales. En suma, se observa un proceso de transición desde un modelo basado en los elementos tangibles de una ciudad hacia otro más preocupado en la búsqueda elementos inmateriales.

PALABRAS CLAVE | Marketing urbano, planificación urbana, participación ciudadana, gestión urbana, políticas urbanas.

ABSTRACT | This article reviews different city case studies in terms of their positioning in the global city system. In the empirical section there is a comparative analysis of 37 urban experiences across two periods of time: 1980-1995 which corresponds to the industrial transition, and 1995-2005 which relates to the post-industrial stage. The article concludes that actions relating to urban quality of life and urban marketing are becoming more important than others more related to strategic economic planning. Furthermore actions of a more material nature are giving way to more immaterial ones. As such, a transition process can be observed from a model based on tangible elementos of the city towards one more concerned with the recovery of more intangible assets.

KEY WORDS | Urban marketing, urban planning, citizen participation, urban management, urban policy. 


\section{Introducción}

En la segunda mitad del siglo XX, el proceso de globalización trajo consigo un incremento de la competencia entre las distintas ciudades. Competencia que se materializa en el intento de mejorar la satisfacción de las necesidades de sus distintos públicos objetivos. Así, cada vez resulta más complicado conseguir la localización de nuevas empresas, captar fondos para mejorar las infraestructuras, conseguir la organización de acontecimientos culturales o deportivos, mejorar la calidad de vida de los habitantes de la ciudad y satisfacer las exigencias de los visitantes. Para lograrlo fueron muchas las ciudades que adoptaron el modelo de la planificación estratégica, principalmente a partir de los años noventa.

Pero, en la práctica, sus objetivos no siempre fueron alcanzados, y cuando lo lograron casi siempre se mostraron insuficientes, porque la globalización introdujo un nuevo escenario de posicionamiento. Como consecuencia, las actuaciones urbanas que debían realizar las instituciones responsables de satisfacer las necesidades de los públicos objetivos de una ciudad fueron cada vez más numerosas y sofisticadas y, por lo tanto, se requerían nuevas herramientas teóricas. En este orden de cosas, los instrumentos típicos de la planificación estratégica se mostraron insuficientes para poder afrontar los nuevos retos del siglo XXI. Es necesario, por eso, buscar nuevas estrategias, más participativas y más innovadoras, que partiendo de "la visión" con una nueva perspectiva global, puedan diseñar acciones estratégicas para su posicionamiento e internacionalización. Es, por ello, el tiempo del marketing urbano.

El marketing urbano es, como se sabe, una disciplina relativamente joven, pero que ha desarrollado rápidamente un armazón teórico (Ashworth, 1993; Ashworth y Voogd, 1990; Berg, Braun y Otgaar, 2002; Chacón y Silva, 2005; D’Orsi, 2005; Elizagarate, 1996, 2003, 2008; Fernández, 2004, 2006; Fernández y Leva, 2004; Fernández y Paz, 2005a, 2005b; Fernández Güell, 1997, 2006; Friedmann, 2004, 2005; Friedmann y Fernández, 2007; Green, 2005; Hall, 1993; Leva, 2004; Martínez, 2004; Metaxas, 2003; Muñiz, 2009; Noissete y Vallerugo, 1996; Paddison, 1993; Pancorbo, 2006; Pancorbo y Hevia, 2004; Paz, 2004, 2006; Precedo, 1996, 2004; Rodell, 1993; Sánchez y Moura, 2005; Seisdedos, 2007; Yao, Sun y Yao, 2005).

A partir de ese cuerpo de conocimientos teóricos se han realizado numerosos trabajos empíricos cuyo objeto de investigación era el marketing urbano: algunos centrados en el campo de la comunicación (Benko, 2000) y (Seisdedos, 1999), otros en la relación con la política industrial y el patrimonio cultural (Álvarez, 2005), otros en el denominado turismo académico (Cubillo y Sánchez, 2005), otros en la influencia de las políticas locales en el ambiente de negocio (Blume, 2006), otros se centran en analizar las claves de éxito en el marketing de una ciudad (Bornemeyer y Rawding, 1994), o en estudiar la influencia de un acontecimiento internacional como vehículo de atracción de una ciudad (Broudehoux, 2007), o en el turismo (Dahles, 1998) y (Limburg, 1998), o en el estudio de los planes estratégicos de marketing en las ciudades alemanas (Fernández y Friedmann, 2007; Franz, 2000). Algunos trabajos empíricos están centrados únicamente en una ciudad, como es 
el caso de Bilbao (González, 2006), Río de Janeiro (Iwata y del Río, 2004), Amsterdam (Kavaratzis y Asworth, 2007), Glasgow (Reason y García, 2007), Londres (Thornley, 1998), Puebla de Los Ángeles, (Domínguez y Bernard, 2005) y Medellín (Fajardo, 2005), mientras otros centran su objeto de investigación en analizar las experiencias urbanas en un grupo de ciudades: ciudades americanas (Meyer, 1979) y ciudades mexicanas y colombianas (Anguiano, 2005). En España no hay muchos trabajos empíricos que contengan una propuesta de marketing urbano para una determinada región geográfica. En este orden de cosas, quizás un trabajo pionero sea la propuesta realizada para el área metropolitana de A Coruña (Precedo, Orosa y Míguez, 2007, 2010), en el que, partiendo de la visión como punto de arranque del proceso, concluye en un modelo de marketing participativo compuesto por doce subproyectos urbanos, sobre los cuales se aplica una metodología de cocreatividad basada en el empowerment, para el diseño final del producto-ciudad.

Desde esta perspectiva, el objeto de investigación de este trabajo es analizar la evolución que han experimentado los contenidos de los programas aplicados en las distintas ciudades, considerado de éxito a lo largo del periodo 1980-2005. Las fuentes utilizadas han sido los informes recopilados por la Asociación Internacional de Planificadores Regionales y Urbanos y por la Asociación Bilbao Metrópoli, tras su posterior estudio pormenorizado mediante el examen de los documentos específicos. Para ello, se han tenido en cuenta los objetivos estratégicos que conformaban dichos contenidos. A continuación, procedemos a estudiar y clasificar la naturaleza de los objetivos identificados para un total de 37 ciudades en el periodo objeto de estudio, correspondiendo 20 a la primera etapa (1980-1995) y 17 ciudades a la segunda (1995-2005). A partir de aquí, organizamos el trabajo en dos apartados: un marco teórico y un análisis empírico; finalmente, se formularon las conclusiones extraídas de la investigación.

\section{1- Las ciudades quieren posicionarse en el nuevo mapa global}

Hasta el comienzo de la década de los ochenta las ciudades tenían como únicas preocupaciones urbanísticas la regulación del crecimiento urbano -mediante el planeamiento urbanístico y territorial-, la rehabilitación y renovación de las áreas centrales y la gestión de áreas metropolitanas cada vez más extensas. A partir de la crisis de la etapa industrial (crisis de la energía de 1973) se produjo una alteración tan significativa, que tanto el sistema productivo como el sistema urbano entraron en una fase de reestructuración. Esto obligó a muchas ciudades a poner en marcha nuevas políticas económicas, primero sociales relativas al empleo, y después las de renovación urbana que sirvieron para ajustar las estructuras heredadas al nuevo sistema productivo, basado en la terciarización de las economías urbanas. En muchas ciudades, llamativas actuaciones de renovación física de los centros se orientaron a recuperar posiciones anteriores o a mejorar su posicionamiento en el nuevo sistema de ciudades. Unas lo lograron, otras no, y fueron aquéllas las nuevas ciudades emergentes, las que sirvieron como referencia para un marco de competitividad urbana en el que las ciudades 
comenzaron a representar el protagonismo de la nueva sociedad. Fueron ocho los componentes que resumían los síntomas de un nuevo diagnóstico urbano: "la ruptura de la relación lineal entre crecimiento de la población, dinamismo y jerarquía urbana; la reestructuración del mercado de trabajo y la formación de bolsas de paro y pobreza urbana; la reducción de la natalidad y del crecimiento vegetativo endógeno sustituido por la creciente aportación inmigratoria; el trasvase sectorial del secundario -la industria - al terciario productivo y al cuaternario especializado y tecnológico; el incremento de las rentas familiares y aumento del tiempo libre con nuevas demandas de ocio; los cambios en la movilidad urbana y la internacionalización de las economías locales" (Precedo, 1996). Un cuadro que volvió a ponerse de manifiesto en estos últimos años, cuando una crisis global estructural provocó una ruptura del ciclo alcista anterior. Ahora, las nuevas tecnologías y las actividades de innovación y la deslocalización empresarial, entonces menos generalizadas, han adquirido mayor protagonismo. La respuesta de las ciudades a la crisis industrial inicial fue muy diferente: "las grandes metrópolis se especializaron como centros de decisiones y de innovación, absorbiendo una proporción importante del nuevo capital social; muchas de las grandes ciudades industriales entraron en declive estructural; algunas ciudades medias se transformaron en polos tecnológicos y del conocimiento, y las actividades de ocio aportaron un fuerte impulso a un número importante de ciudades de todo el mundo" (Precedo, 1996).

En la figura siguiente resumimos la evolución experimentada por las políticas urbanas, tanto económicas como territoriales, en esa sucesión de ciclos y puntos de ruptura.

FIGURA 1 | Evolución de las políticas urbanas

\begin{tabular}{|c|c|c|c|c|}
\hline & & INDUSTRIAL & POSTINDUSTRIAL & GLOBALIZACIÓN \\
\hline $\begin{array}{r}\text { ACTIVIDADES } \\
\text { MOTRICES }\end{array}$ & $>$ & $\begin{array}{l}\text { Industrial terciario } \\
\text { comercial }\end{array}$ & $\begin{array}{l}\text { - Terciario avanzado } \\
\text { - Sistema financiero } \\
\text { - Comunicaciones }\end{array}$ & $\begin{array}{l}\text { - Nueva economía } \\
\text { - Tiempo libre } \\
\text { - Cultura } \\
\text { - Formación } \\
\text { - Salud } \\
\text { - Oficinas globales }\end{array}$ \\
\hline FORMA URBANA & - & $\begin{array}{l}\text { Áreas metropolitanas } \\
\text { con gravitación central }\end{array}$ & Red urbana dispersa & $\begin{array}{l}\text { Red Urbana } \\
\text { Policéntrica }\end{array}$ \\
\hline $\begin{array}{r}\text { POLÍ́TICA } \\
\text { URBANA }\end{array}$ & 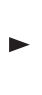 & $\begin{array}{l}\text { Equidad (zonning, des- } \\
\text { equilibrios, segregación, } \\
\text { sociedad del bienestar) }\end{array}$ & $\begin{array}{l}\text { Mercado (gestión público- } \\
\text { privado, dirección, } \\
\text { competitividad) }\end{array}$ & $\begin{array}{l}\text { - Calidad urbana } \\
\text { - Atractividad } \\
\text { - Internacionalización }\end{array}$ \\
\hline PLANIFICACIÓN & 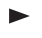 & Planes de urbanismo & Planificación estratégica & Marketing urbano \\
\hline GOBERNABILIDAD & $\longrightarrow$ & Tecnoestructura pública & Asociacionismo público & $\begin{array}{l}\text { - Control ciudadano } \\
\text { - Participación social }\end{array}$ \\
\hline
\end{tabular}

FUENTE ELABORACIÓN PROPIA. 
Pero, en este complejo contexto, unas ciudades lograron importantes avances que hicieron de ellas ciudades de referencia en la mayoría de los estudios, y que son las que consideramos emergentes; en ellas basamos el análisis empírico que presentamos. En cambio, otras muchas ciudades o no quisieron o no supieron posicionarse. Hemos diferenciado tres fases evolutivas, dos correspondientes al paradigma transicional industrial-postindustrial, y una tercera al postindustrial propiamente dicho.

\section{2- Las ciudades pioneras: los antecedentes de la nueva política urbana}

Las ciudades industriales más afectadas por la crisis, genéticamente heredera de la primera revolución industrial, aplicaron como modelo una primera versión de la planificación estratégica empresarial, muy centrada en el empleo. Sin embargo, ésta no siempre llegó a dar el resultado esperado, lo cual motivó una segunda generación de planes estratégicos basados en la ecuación investigación-cultura-tecnología (modelo Pittsburg) asociados, en su mayoría, a grandes operaciones de cambio de imagen mediante la renovación urbana y la rehabilitación y revitalización de los centros (modelo francés, como el de Burdeos). En algunas se incorporó un modelo inicial de marketing urbano, generalmente asociado a la arquitectura-escaparate, con la intención de proyectar la ciudad en el exterior y así poder captar nuevas inversiones y empresas, lo cual casi nunca se consiguió en la práctica; o para atraer más visitantes, porque todas vieron en el turismo un nuevo motor para el desarrollo urbano y para su terciarización. Pero, a corto plazo, más éxito que la planificación estratégica urbana aportaron las operaciones derivadas de la celebración de grandes eventos mundiales o internacionales (Olimpiadas, Exposiciones) y el desarrollo de la cultura como un nuevo campo de apoyo a la competitividad urbana. Un caso significativo, aunque más tardío, fue el de Barcelona, tradicionalmente la capital industrial y económica de España, pero también afectada por la crisis industrial. Tras varios proyectos fallidos, compitió con éxito para la celebración de las Olimpiadas de 1992, una oportunidad estratégica que justificó un importante plan de renovación urbana. Previamente se había puesto en marcha un excelente plan de marketing ciudadano para implicar a la población y a las instituciones en el acontecimiento; después, se diseñó la operación titulada "abrir la ciudad al mar", una marca que fue emulada por otras muchas ciudades españolas. Esta operación logró crear una gran fachada marítima como espacio de ocio y turismo y reforzar la imagen de ciudad mediterránea. Otras operaciones posteriores de marketing cultural no tuvieron el éxito esperado (el Fórum mundial) y la ciudad sólo ha logrado preeminencia como gran destino del turismo internacional, pero ha perdido peso económico y cultural en el contexto del sistema español y europeo de ciudades.

Valorizadas por el efecto promocional que aporta la innovación, algunas ciudades portuarias fueron pioneras aplicando el altamente difundido modelo del waterfront, basado en la recuperación de los frentes marítimos y espacios portuarios obsoletos adyacentes, mediante complejas operaciones de renovación urbana, con el fin de crear centros de ocio, de residencia y terciarios de calidad sobre los 
antiguos terrenos que la evolución del transporte marítimo había dejado sin uso. Rotterdam fue pionera en Europa, siendo también allí, en su Universidad, donde se creó el concepto de marketing urbano (Leo van der Berg). En Estados Unidos, con un modelo sencillo, Baltimore realizó un buen trabajo de reestructuración portuaria e industrial con fines turísticos. Este primer modelo fue seguido por muchas otras ciudades portuarias de la revolución industrial, con diversas variantes y resultados desiguales (Amberes, Hamburgo, Cardiff, Liverpool) o por ciudades portuarias desactivadas como Génova o Trieste. La operación de mayor dimensión fue la de los Docklands de Londres. La denominación genérica de waterfront se extendió también a frentes fluviales y a ciudades balnearias del litoral (talasoterapia y ocio). Entre las ciudades industriales en crisis, destacaron los logros de Lille, Birmingham, y en especial Glasgow, como caso experimental de reestructuración, pero no llegaron a alcanzar las posiciones anteriores.

Mientras tanto, la experiencia empírica demostró que la trilogía servicios-ociofinanzas, con base en su sistema productivo local internacionalizado, se reveló en la práctica como la fórmula más eficaz.

\section{1 - Las ciudades de primera generación}

El nuevo modelo de desarrollo y renovación urbana surgido de las experiencias anteriores dio paso a una primera generación de ciudades emergentes, que con una perspectiva estratégica y una metodología más integradora -menos preocupada por las debilidades y más por las oportunidades que sus puntos fuertes o ventajas comparativas les otorgaban- pusieron en marcha políticas urbanas más innovadoras. Probablemente, cinco fueron las ciudades que entraron en el exclusivo club de ciudades de referencia, cuatro del mundo más desarrollado y una perteneciente al mundo subdesarrollado.

De las ciudades emergentes de primera generación, la primera fue Sydney, que promovió un novedoso modelo de ciudad verde sostenible y una mutación de la actitud social de sus ciudadanos que se convirtió en principal activo, para ser una ciudad "innovadora, cosmopolita y abierta". El símbolo necesario para la nueva ciudad se concretó en el Teatro de la Ópera, convertido en uno de los emblemas urbanos de la ciudad contemporánea, y el primer caso que utilizó la arquitectura como operación de marketing urbano.

También Boston, que atravesaba una etapa de decadencia económica, apostó por un buen urbanismo para hacer más competitiva la ciudad, aunque esta idea inicial evolucionó hacia el potencial de conocimiento que la ciudad acumulaba. En efecto, su área metropolitana tiene 101 municipios, y en ella está la famosa Ruta 128 , el principal centro mundial de alta tecnología y conocimiento en un contexto universitario (Harvard, M.I.T. y otras universidades y laboratorios), al que se añadieron innovadoras iniciativas tecnológicas, de modo que su enorme potencial de conocimiento se sumó al sector financiero local, a la medicina y la investigación biomédica y al desarrollo alcanzado en las nuevas tecnologías. Todo este programa de desarrollo del conocimiento se acompañó de otro programa de parques urba- 
nos muy innovador, como imagen verde de ese nuevo modelo de estilo de vida que debería acompañar a la calidad y excelencia del desarrollo urbano. Se acometieron también interesantes actuaciones de recuperación de edificios portuarios para actividades de ocio y se renovaron las áreas portuarias obsoletas para nuevos usos. Todo ello, unido al plan metropolitano, situaron a Boston en una posición más competitiva, de la cual el mejor exponente fue el Big Dig, la mayor inversión en una infraestructura urbana acometida en Norteamérica. Se trataba de soterrar la principal arteria de tráfico urbano para crear, en el contacto con el puerto renovado, un espacio abierto que sirviera como atractivo al nuevo capital intelectual.

La tercera ciudad, Dublín, también basó en el capital humano y en el conocimiento su programa de desarrollo urbano. La capital de un país que había centrado su espectacular crecimiento económico en la educación y en la tecnología, precisaba un nuevo modelo de ciudad adaptado a la nueva Irlanda. Tomando como punto fuerte la cooperación entre el sector público, el empresarial y el social, la ciudad se convirtió en un referente en la industria electrónica y en servicios financieros internacionales. Para ello fue necesario acometer un ambicioso programa de renovación urbana, que incluía el transporte en metro, la delimitación de áreas de regeneración física y social, la regeneración de la arteria central histórica como espacio de ocio, cultura y turismo o la reutilización de los edificios portuarios para centros financieros y de servicios empresariales internacionales. Todo esto, más el Distrito Digital, hicieron que Dublín reforzase su posición estratégica global, aun cuando la crisis actual debilitó considerablemente el modelo.

El cuarto caso es el de Toronto. La ciudad del sur de Canadá basó su modelo de posicionamiento estratégico en la integración de la elevada población inmigrante en el tejido urbano, económico y social de la ciudad, junto con la regeneración de un frente litoral de $300 \mathrm{~km}$.: Lake Ontario Waterfront. Las largas sendas peatonales y de bicicletas, la mejora ambiental y paisajística y la sostenibilidad de la economía urbana fueron acciones emblemáticas de acompañamiento. Fue en esta misma línea, pero adaptada a sus características, en donde descansa el éxito de una ciudad brasileña pionera en el desarrollo sostenible: Curitiba. El hoy gran centro de la industria automovilística, atrajo a las inversiones empresariales a partir del prestigio urbano que le otorgó su plan de desarrollo urbano sostenible. La clave en este caso fue la educación ambiental activa, la participación ciudadana, el sistema original e innovador de transporte colectivo, el sistema de parques y su mantenimiento, el sistema de gestión de residuos y la estrategia metropolitana de suelo. La ciudad del "Cambio Verde" es hoy la capital ecológica de Brasil, y una de las ciudades más innovadoras del mundo, razón por la cual compañías industriales y de servicios internacionales buscaron localizarse allí.

Estas cinco ciudades representan la contrapartida de los modelos de desarrollo urbano ligados a infraestructuras y a los planes estratégicos de primera generación, excesivamente economicistas y con una metodología rígida e insuficiente. Todas ellas trataron de dar una apuesta alternativa con estrategias territoriales basadas en el conocimiento y en la formación de capital social como objetivos preferentes. 
Principios o ejes estratégicos que marcaron una referencia innovadora para otras muchas ciudades que iniciaron después el diseño del camino hacia el futuro, lógicamente, cada una adaptándolo a sus características.

\section{2- Las ciudades de segunda generación}

Una segunda generación de ciudades de éxito se puede agrupar en torno a las que, tras las adaptaciones estructurales y morfológicas efectuadas, basaron su estrategia de posicionamiento en el marketing urbano. Fueron estrategias urbanas que nos sirvieron para saber cómo adaptar estructuras urbanas antiguas al presente postindustrial, o cómo alcanzar un nuevo punto de equilibrio sobre el que proyectar una imagen de futuro en el nuevo marco de la sociedad del conocimiento.

En la ciudad inglesa de Newcastle, la recuperación de su frente fluvial fue la actuación clave, utilizando para el marketing los puentes y el Centro de Arte Contemporáneo construido sobre un antiguo silo portuario. La ciudad americana de Cleveland era otra ciudad industrial en crisis, con importantes áreas de marginalidad, criminalidad y conflictividad social. El voluntariado universitario como estrategia de integración y participación en la nueva ciudad, además de la adaptación del tejido productivo y comercial a la nueva economía, sirvieron para construir el plan de marketing: "hacer lo que sabían hacer pero de otra manera". La recuperación del frente del lago y la renovación urbana tuvieron en el Rock and Roll Hall of Fame and Museum su señal de identidad, en la que basaron una estrategia de marketing que utilizó lo antiguo adaptándolo a la nueva sociedad urbana y tecnológica. Una eficaz concreción de la marca diseñada.

Más significativo fue el éxito de Pittsburg, también en Estados Unidos. El plan se inició en 1950. La primera fase se centró en la renovación del centro, haciendo de él un hermoso downtown; la siguiente, en las grandes infraestructuras y la posterior, la del conocimiento, en la creación de uno de los centros de formación en dirección y gestión de empresas más cualificados del mundo, que funciona como centro de atracción de estudiantes, empresarios e inversores. Fueron muchas las empresas que se crearon bajo la inspiración de universidades muy avanzadas u optaron por localizarse en ese nuevo entorno de prestigio, haciendo que una vieja ciudad industrial en crisis sea hoy una de las ciudades más gratas para vivir de Estados Unidos. Las multinacionales convencionales del acero descentralizaron sus fábricas a países iberoamericanos. La ciudad acuñó un modelo propio de proyecto-ciudad que se convirtió en un producto de marketing, al que se asociaron otras ciudades interesadas en recibir sus enseñanzas, formando "una red de aprendizaje".

La clave de estos programas reside en que en todas las ciudades había un entorno innovador del que supieron sacar partido, acudiendo más a la imaginación y a la creatividad -a lo inmaterial en definitiva- que a la técnica, demostrando el valor de lo intangible en la nueva generación de proyectos urbanos.

Pero, tal vez, el caso más representativo sea el que también alcanzó el mayor éxito del grupo: la ciudad americana de Seattle, declarada la mejor ciudad de negocios, con un aeropuerto que es líder mundial en transporte intermodal, y con 
90.000 personas trabajando en los nuevos sectores industriales de la aeronáutica, la electrónica y la robótica (Microsoft). La ciudad en la que nació Bill Gates supo hacer de este hecho un argumento para valorizar su condición de entorno innovador, un valor incuestionable con un razonamiento lógico muy bien planteado. Un sector de rascacielos con firmas de importantes arquitectos, compusieron la imagen renovada de la vieja ciudad, popularizándola en todo el mundo a través del cine y la televisión, al convertirse en un escenario familiar para todos nosotros. Hoy figura en lugares destacados en todos los rankings sobre calidad de vida urbana, lo cual se ha convertido en un valor añadido de competitividad.

En este grupo de ciudades podríamos incluir también la ciudad española de Bilbao. Su área metropolitana había sufrido los efectos de la crisis industrial, dejando en torno a lo que fue el eje fluvial industrial y portuario anterior un escenario de decadencia. Un largo proceso de reflexión y planificación estratégica fue poniendo en valor los diferentes elementos heredados, siguiendo la secuencia siguiente: primero se adoptó la idea de convertir la "ría en una nueva avenida fluvial de la ciudad postindustrial"; después se diseñó un ambicioso plan de renovación urbana del frente portuario e industrial obsoleto y vacante y, finalmente, se propugnó una nueva imagen de marca para posicionar la ciudad en el mapa de ciudades innovadoras a partir de la arquitectura escaparate. El museo diseñado por Frank Gehry se convirtió en un icono global del final del siglo XX. El éxito del proyecto hizo que el apelativo "efecto Gugemheim" se aplicara a los proyectos de marketing urbano que tomaron un gran centro museístico o cultural como estrategia de posicionamiento. La realidad es que, aparte de la renovación urbana, la atractividad turística y el valor de posicionamiento, los demás efectos esperados no se produjeron y la ciudad vasca no logró recuperar su función económica anterior ni captar los activos tecnológicos esperados.

\section{3- Las nuevas ciudades del siglo XXI: la ciudad de marketing}

Hemos visto ciudades que adaptaron el pasado al presente, otras que buscaron en sus puntos fuertes apoyos para nuevas estrategias de competitividad, en el contexto de la sociedad del conocimiento, y muchas que hicieron de la renovación urbana y de la arquitectura de marketing un emblema de posicionamiento en el nuevo sistema de ciudades. Pero, además de éstos, está surgiendo un nuevo grupo: el de las ciudades que tienen el propósito de ser las capitales innovadoras del siglo XXI, y ya hay tres que están perfectamente situadas, Singapur, Dubai y Abu Dhabi. En todos los casos se trata de un proyecto de marketing internacional.

La primera es una ciudad estado, situada en una isla, con 3,5 millones de habitantes chinos, malayos e hindúes, donde todos hablan inglés. Singapur, un centro del contrabando, de la droga, de la violencia y del crimen se transforma para construir el "país ideal de nuestros nietos". Esta ciudad-nación es hoy el gran centro de negocios, de servicios avanzados y de congresos del sudeste asiático, con universidades, sanidad y comunicaciones muy competitivas. En el paso de una ciudad del crimen a uno de los centros de negocios más seguros del mundo, gracias a la participación de 
sus profesionales más cualificados, hay todo un itinerario de transformación urbana. Hoy es el puerto y el aeropuerto más eficiente del mundo, y su población es la de mayor incorporación a la sociedad del conocimiento y a las nuevas tecnologías, es decir, el marco de la ciudad del siglo XXI, aunque algunos excesos tecnológicos pueden dar lugar a debates antropológicos interesantes, aunque serían debates de futuro.

Dubai y Abu Dhabi son dos ciudades pertenecientes a los Emiratos Árabes Unidos. Se han construido en muy poco tiempo gracias a la generosa financiación proporcionada por el petróleo. Dubai no sobrepasa los 900.000 habitantes, pero se ha convertido en el centro de negocios del mundo islámico para los occidentales, posicionándose a nivel mundial como centro de turismo de lujo y exponente del marketing urbano. Su situación ventajosa, la construcción de infraestructuras de primer orden: puerto y aeropuerto internacional y un perfil arquitectónico de vanguardia hacen de Dubai una ciudad excepcional. Una de sus islas artificiales, The Palm, alberga un proyecto inmobiliario en el que han comprado muchos millonarios europeos, provocando que otros proyectos semejantes empezaran a ponerse en marcha. Su hotel de siete estrellas, el Burj al Arrab y el edificio más alto del mundo recientemente finalizado, Burj Tower, son los símbolos del grandioso proyecto de citymarketing. Sin embargo, y en contra de las expectativas creadas, la crisis global ha puesto en evidencia importantes debilidades en un proyecto banal y asentado en futuribles. En este mismo orden de cosas, Abu Dhabi intenta convertirse en un centro de ocio a escala mundial. Para eso, cuenta con proyectos como: el nuevo Museo del Louvre, el más grande de los museos Guggenheim -también ideado por Frank Gehry- y un Centro de Artes Interpretativas (Muñiz, 2009), además del parque de atracciones de Ferrari. Aunque de momento sólo es un gran proyecto.

En esta línea de proyección urbana, aunque a una escala inferior, puede mencionarse la ciudad española de Valencia. Su débil posicionamiento en la jerarquía urbana española motivó la puesta en marcha de un plan estratégico encaminado a lograr la preeminencia portuaria en el Mediterráneo, y convertirse en un gran centro de ocio con grandes eventos centrados en el automovilismo y la vela. Además, la nueva marca-ciudad se asoció a la firma del arquitecto local Calatrava, quien construyó un discutido centro cultual y de ocio denominado Ciudad de las Artes y las Ciencias, unido a un gran centro internacional de negocios.

Fue así como a los casos pioneros se sumaron las ciudades emergentes de la primera y segunda generación, con proyectos más estructurados y polivalentes, que con las nuevas ciudades globales del siglo XXI dibujan un nuevo mapa urbano mundial, en el que cada vez más ciudades tratan de encontrar su sitio, al igual que otras lo hicieron antes, aunque con diferente suerte y acierto cada una.

\section{4- La ciudad del siglo XXI como problema: las ciudades medias internacionales como alternativa}

Una visión de la sociedad urbana actual y del futuro basada sólo en las ciudades de éxito constituiría una visión deformada de la realidad. Una realidad que nos dice que en el mundo existen hoy más de 500 aglomeraciones urbanas con más de un 
millón de habitantes, y que la mayoría de ellas y las que más crecen pertenecen a países subdesarrollados o en vías de desarrollo. En ellas la urbanización creciente es una manifestación de la falta de oportunidades que tienen abultadas poblaciones rurales, las cuales escapan hacia la ciudad para convivir con la pobreza de la que sólo los más afortunados podrán salir. Tampoco en las ciudades desarrolladas las cosas son todas positivas, ya que en ellas las bolsas de pobreza aumentan a razón que lo hace el desempleo, la deslocalización, la marginalidad y la inmigración, creando ese cuarto mundo de pobres en el corazón de los países ricos. Pero no sólo es esto, sino que también el terrorismo, la inseguridad y la integración de una inmigración internacional masiva, son hoy una amenaza para todas las ciudades; así, también son problemas de difícil resolución el transporte urbano, el envejecimiento demográfico de los centros, la vivienda, la contaminación, la energía y el agua.

Por esto al grupo selecto de ciudades emergentes hay que añadir el de las que lograron resolver sus problemas, haciendo que, frente a las brillantes políticas estratégicas neoliberales - muchas veces más orientadas a reforzar el liderazgo político que a dar respuesta a los problemas reales de la mayoría - haya que considerar que en la sociedad urbana del Tercer Milenio serán las políticas sociales las que demanden el mayor interés. Al menos, si se quiere que sea la persona, el ser humano, el centro de una civilización que debe cambiar el modelo de éxito y la imagen por una verdadera sostenibilidad, solidaridad y cooperación, más allá de lo que sofisticados y repetitivos planes estratégicos presentan como declaraciones de principios, éstas deben transformarse en valores sobre los cuales puedan construirse verdaderas Comunidades Territoriales y Urbanas diseñadas desde los mecanismos psicosociales que regulan la conducta humana, haciendo de la cohesión social un activo preferente para alcanzar el estatus de ciudad innovadora y competitiva (Precedo, 2004). Tal vez haya llegado el momento de plantear las cosas de otra manera; pero, por ahora, las ciudades emergentes son las ciudades de éxito en el contexto de un mercado que encuentra en la competencia entre ciudades una justificación más teórica que real, y que al final nos dibujan un hipotético mercadeo de ciudades en que todas quieren competir en el mismo segmento y por los mismos objetivos. Quizás sea esta falta de diferenciación lo que haga que los resultados estén más en el papel y en la apariencia que en la realidad profunda de las ciudades mismas.

Una idea alternativa o complementaria que subyace es que también las Ciudades Medias de rango internacional pueden posicionarse en el sistema de ciudades globales, como centros de atractividad basados en la calidad de vida urbana como su principal ventaja competitiva.

En efecto, así como inicialmente se impuso la tesis de Castells (Castells, 1996) de que los nodos de la economía global estaban territorialmente concentrados en las grande áreas metropolitanas mundiales, a medida que el proceso de globalización se fue difundiendo a más territorios, surgió un nuevo planteamiento de las ciudades globales, siendo "al final la globalización de las empresas y las nuevas tecnologías de la información lo que marca la importancia de las ciudades” (Sassen, 1991, 2007). Más recientemente, Peter Hall (Hall, 2001) definió las ciudades mun- 
diales en términos de "múltiples roles" o funciones, la mayoría correspondientes al terciario avanzado. También utiliza el concepto de jerarquía global de ciudades, que había introducido John Friedmann unos años antes. El resultado fue que frente a la tesis de las grandes metrópolis de Castells, el proceso de difusión ha generado una nueva jerarquía de ciudades globales, es decir, ciudades internacionales que constituyen nodos de servicios especializados. De este modo, junto a las grandes ciudades globales, Peter Hall introdujo el concepto de ciudades subglobales, que "además del terciario económico especializado atraen actividades de turismo y ocio apoyadas en su reputación cultural". Una nueva jerarquía urbana, en la que la creciente ampliación de los espacios de la globalización permite pensar que algunas Ciudades Medias, puedan incorporarse al tercer escalón de la nueva jerarquía urbana, como nuevas centralidades globales intermedias.

Este es un modelo de atractividad en el que, además de los factores socioeconómicos y urbanísticos, se empieza a considerar como estrategia de posicionamiento la promoción de los recursos endógenos que el área metropolitana posee, superando el proceso de crecimiento cuantitativo basado en la acumulación capitalista de recursos, sustituyéndolo por otro de valorización cultural y social. Un modelo que, por un lado, reinterpreta el significado ideográfico de los espacios públicos a partir de una reinvención del pasado y, por otro, aporta una visión sobre la cual apoyar propuestas estratégicas de marketing urbano basadas en la creatividad social (Precedo, Orosa y Míguez, 2010). En todo caso, el avance de la planificación participativa y la nueva consideración del empowerment como mecanismo de devolución del poder a la sociedad civil para la construcción de la ciudad real, abre un interesante camino a la planificación estratégica y, particularmente, al marketing ciudadano, alejado y distinto de un proceso participativo meramente institucional y anclado en los detentadores del poder social, económico y político.

\section{3- Análisis empírico: la evolución de los objetivos de las políticas de renovación y de competitividad urbana}

En esta segunda parte, vamos a descender del nivel de generalización a la praxis urbana, analizando la evolución de los objetivos y las actuaciones más significativas que forman parte de los planes que se han llevado a cabo en un conjunto de treinta y siete ciudades que han logrado posicionarse como ciudades de éxito o de buenas prácticas y que por eso suelen ser tomadas como referencia, muchas de las cuales hemos enumerado en el apartado anterior ${ }^{1}$. A ellas hemos agregado las ciudades españolas seleccionadas entre las más innovadoras, entre las cuales incluimos las tres a las que hemos hecho mención. Se trata, en definitiva, de constatar empíricamente las conclusiones obtenidas en el estudio de casos que hemos hecho, para darles mayor objetividad. En total hemos estudiado 37 ciudades y en ellas identificamos un

1 Sydney, Boston, Dublin, Toronto, Curitiba, Newcastle, Pittsburg, Seattle, Rotterdam, Baltimore, Amberes, Hamburgo, Cardiff, Liverpool, Génova, Trieste, Lille, Birmingham, Glasgow y Cleveland. Las españolas: Madrid, Barcelona, Valencia, Sevilla, Bilbao, Alicante, Pamplona, Zaragoza, Vitoria, Oviedo, Gijón, La Coruña, Vigo, Málaga, Santiago de Compostela y Palma de Mallorca. 
total de 280 actuaciones urbanas, es decir, las que han sido ejecutadas entre las previstas en los períodos de tiempo seleccionados. Para el análisis comparativo hemos agrupado las ciudades en dos clases:

1 Los proyectos urbanos correspondientes a la primera etapa de la transición postindustrial, que iniciaron y cumplieron la mayor parte de sus objetivos entre 1980 y 1995 , y que totalizan 124 actuaciones.

2 Las ciudades que empezaron a desarrollar su proyecto urbano entre $1995 \mathrm{y}$ 2005, y que suman 156 actuaciones urbanas.

La metodología del análisis evolutivo fue la siguiente: clasificamos los tipos de actuaciones que se han llevado a cabo en cada etapa y, después, mediante un estudio comparativo, obtuvimos los tipos de acciones que han tenido una tendencia positiva y las que, al perder peso relativo, ofrecen una tendencia negativa.

\subsection{Las ciudades innovadoras de la transición: las acciones estratégicas de ajuste}

Se incluyen aquí las ciudades que definieron un plan de ajuste sustitutivo del modelo industrial anterior para adaptarlo a la transición al modelo postindustrial. Se trata, en casi todos los casos, de ciudades portuarias y manufactureras, que precisaban adaptarse a la nueva realidad urbana. El proyecto básico tenía una doble orientación: la mejora física de los centros (rehabilitación morfológica) y de los espacios obsoletos (renovación urbana), y la planificación estratégica, orientada a recuperar o dinamizar la competitividad urbana en términos económicos (planificación estratégica económica), de acuerdo con los modelos teóricos antes expuestos.

CUADRO 1 Actuaciones urbanas innovadoras en la etapa de transición

\begin{tabular}{lc}
\hline Tipo de ACTUACiOnes & $\begin{array}{c}\text { Peso Relativo De CADA UNA } \\
\text { SOBRE EL TOTAL (\%) }\end{array}$ \\
\hline Renovación morfológica & 18,55 \\
Atractivo empresarial & 17,74 \\
Infraestructuras & 14,52 \\
Turismo y ocio & 11,29 \\
Imagen y marca & 6,45 \\
Medio ambiente y sostenibilidad & 5,65 \\
Calidad de vida residencial & 4,84 \\
Cultura & 4,03 \\
Actividades de conocimiento & 3,23 \\
Nueva economía & 3,23 \\
Cooperación capital privado & 3,23 \\
Eventos & 2,42 \\
Arquitectura de marketing & 2,42 \\
Integración social & 1,61 \\
Participación & 0,81 \\
Totales & 100,00 \\
\hline
\end{tabular}

FUENTE ELABORACIÓN PROPIA. 
a El primer lugar, por número de actuaciones y de acuerdo con el modelo, correspondió a las acciones de renovación morfológica. Se aprovecharon los espacios industriales portuarios y en desuso o vacantes como una oportunidad para mejorar el atractivo de la ciudad, y como refuerzo a las acciones de promoción urbana. La mayoría de las intervenciones inventariadas se refieren a la rehabilitación, renovación y revitalización de los centros históricos, que habían alcanzado un grado de deterioro notable. Junto con esto, destacan las actuaciones en los frentes marítimos, especialmente de los antiguos espacios portuarios, para adaptarlos a nuevos usos relacionados con el nuevo modelo de ciudad. En ellos se instalarán los nuevos centros de dinamización cultural y económica que se promovieron a través de grandes actuaciones arquitectónicas y urbanísticas. Lógicamente, este tipo de intervenciones incluye la integración de la ciudad y el puerto o la apertura de la ciudad al mar o a los ríos a través del diseño de nuevos frentes fluviales o marítimos. Coincide con el modelo del waterfront.

b El segundo grupo, por orden de importancia, corresponde a los objetivos encaminados a sustituir el modelo de economía urbana industrial por el modelo de la ciudad de servicios o economía terciaria. La mayor parte de las actuaciones examinadas se orientaron a la atracción de sedes de empresas multinacionales y de nuevas actividades de servicios. Para ello, crearon nuevos centros financieros y de negocios internacionales en las áreas centrales desafectadas, que se apoyaron en nuevas infraestructuras de conexión; particularmente, se potenciaron los aeropuertos hacia los negocios, promoviendo enlaces internacionales.

c La tercera posición es para las actuaciones de carácter infraestructural. La mayoría de ellas estuvieron encaminadas a mejorar las condiciones de accesibilidad en el centro de las ciudades, la creación de plataformas intermodales y la conexión con la red viaria exterior, aprovechando también espacios vacantes que ocupaban antiguas actividades portuarias o industriales. También destaca el esfuerzo en priorizar el transporte público, preferentemente los ferrocarriles metropolitanos (incluye el metro ligero y el tranvía). En algunos casos se añadieron nuevas infraestructuras de telecomunicaciones (telepuertos) y centros logísticos.

d El cuarto grupo, por el número de actuaciones, corresponde a las actividades de turismo y ocio localizadas en los centros históricos o en los nuevos frentes urbanos. Todos los planes estratégicos incluían como objetivo prioritario la atracción de visitantes, de modo que todas las ciudades optaron por convertirse en centros turísticos, lo cual, en principio, facilitó la recuperación y revitalización de los centros históricos.

e Agrupamos ahora cuatro tipos de actuaciones de importancia intermedia (entre el 4,0\% y 6,4\%). Todas ellas tuvieron como preocupación común la mejora de la imagen urbanística, la mejora de la calidad medioambiental, la creación de espacios verdes, la mejora de la calidad residencial en las ciudades centrales y la construcción de grandes centros culturales (museos, auditorios y teatros).

f En sexto lugar, incluimos el conjunto de actuaciones con menor prioridad para los proyectos urbanos considerados (entre 2,4\%y 3,2\%). Todas ellas estuvieron 
encaminadas a mejorar la cualificación de los recursos humanos y de las organizaciones empresariales locales, para adaptarlos a las nuevas tecnologías y a los nuevos sectores productivos emergentes. Para ello, se logró una importante -aunque desigual- colaboración del sector privado, que también intervino en las otras dos acciones incluidas en este grupo: la promoción de eventos internacionales, como justificación del proyecto urbano y la captación de fondos, y la construcción de edificios emblemáticos para usos culturales y de ocio.

g Finalmente, fueron muy escasas las acciones encaminadas a mejorar la integración social y la participación ciudadana, lo cual coincide con el modelo teórico que hemos expuesto en el apartado anterior, en el que la gestión de la ciudad correspondía preferentemente a organizaciones de tecnoestructura del sector público, es decir, una política top-down.

\section{2- El proyecto de ciudad innovadora postindustrial: las acciones estratégicas de atractividad y competitividad}

En relación con la etapa anterior se ha producido, como enseguida observaremos, una pérdida de peso de los objetivos estratégicos de carácter económico a favor de la calidad urbana.

CUADRO 2 Actuaciones urbanas en la fase postindustrial

\begin{tabular}{lc}
\hline Tipo de ACTUACiONES & $\begin{array}{c}\text { Peso Relativo De CADA UNA } \\
\text { SOBRE EL TOTAL }(\%)\end{array}$ \\
\hline Renovación morfológica & 13,46 \\
Atractivo empresarial & 12,82 \\
Imagen y marca & 12,18 \\
Infraestructuras & 11,54 \\
Medio ambiente y sostenibilidad & 8,33 \\
Calidad de vida residencial & 7,69 \\
Turismo y ocio & 5,77 \\
Arquitectura de marketing & 5,13 \\
Nueva economía & 4,49 \\
Cultura & 3,85 \\
Actividades de conocimiento & 3,21 \\
Participación & 3,21 \\
Integración social & 3,21 \\
Cooperación capital privado & 3,21 \\
Eventos & 1,92 \\
Totales & 100,00 \\
\hline
\end{tabular}

FUENTE ELABORACIÓN PROPIA.

a Ocupan el primer lugar las operaciones de rehabilitación de áreas residenciales, y aunque la recuperación de nuevos usos de los frentes portuarios sigue siendo relevante, adquiere mayor importancia la renovación de las áreas urbanas en su conjunto y de los barrios de la posguerra, y no sólo de los centros históricos como antes ocurría. 
b El segundo lugar es para los objetivos relacionados con la adaptación del sistema productivo urbano a la nueva economía, para aumentar la atractividad empresarial de la ciudad. Preferentemente estuvieron orientados hacia los centros y servicios logísticos y hacia los centros internacionales de negocios, formando parte todo ello del sistema de servicios avanzados. Ya habían sido planteados en la etapa anterior, pero se desarrollaron en ésta.

c Llama la atención que asciendan al tercer lugar las actuaciones de marketing estratégico urbano, relacionadas con la imagen y marca de la ciudad, y orientadas al posicionamiento exterior del producto-ciudad.

d Las infraestructuras pierden peso, pasando a ocupar un cuarto lugar. Entre ellas, aumentan los sistemas de transporte intermodal, la potenciación del transporte colectivo y, sobre todo, la construcción de redes ferroviarias suburbanas (metros). Siguen estando presentes actuaciones relacionadas con la potenciación de los aeropuertos y las telecomunicaciones.

Tras estos cuatro tipos de intervención que concentran el 50\% de las actuaciones, el $50 \%$ restante se reparte entre once tipos de medidas que podemos agrupar en tres clases:

1 Cinco de estas actuaciones aglutinan el $27,4 \%$ del total y corresponden, por este orden, a: objetivos de sostenibilidad urbana (medio ambiente, zonas verdes y peatonalización); mejora de la calidad de las áreas residenciales de nueva creación; actividades de turismo urbano y ocio, y arquitectura de marketing asociada a las operaciones de cambio de imagen. Quedan finalmente en este grupo objetivos relacionados con actividades de la nueva economía. Un conjunto de actuaciones que define perfectamente cuál es el perfil de la ciudad postindustrial.

2 El siguiente grupo está formado por 5 tipos de intervenciones que apenas suman el $17 \%$ del total. Tienen que ver con la cultura local, las actividades del conocimiento, la participación ciudadana y la integración social, las cuales constituyen el marco definitorio de un nuevo entorno urbano, en donde las políticas sociales empiezan a adquirir peso. Aunque a veces no pasa de ser testimonial, marca un punto de partida para un cambio en el modo de ver la ciudad más allá del enfoque economicista liberal de la planificación estratégica inicial.

3 Queda, finalmente, un tipo de objetivo que, por lo que los datos demuestran, tiene cada vez menos importancia en número, pero mucha en trascendencia. Se trata del modelo de desarrollo urbano basado en la celebración de eventos internacionales.

Esta enumeración constituye un diagnóstico del cambio y verifica la transferencia de políticas infraestructurales o materiales hacia política de calidad e intangibles. Este resultado se matizará mucho mejor al hacer una comparativa. 


\subsection{La evolución de las acciones estratégicas: la valorización de los intangibles}

Tras el análisis descriptivo del tipo de actuaciones, presentamos a continuación los resultados obtenidos del estudio comparativo entre el peso o importancia de cada tipo de acción en cada una de las fases. De ella, se obtiene una doble clasificación:

1 Acciones expansivas: son aquellas que han incrementado su peso o importancia entre las dos fases. Lógicamente, son las que despertaron mayor interés por agrupar acciones consideradas de éxito.

CUADRO 3 | Evolución de las actividades motrices innovadoras en la ciudad industrial (A) y postindustrial (B)

\begin{tabular}{lc}
\hline TIPO DE ACTUACIONES & DIFERENCIA B-A \\
\hline Imagen y marca & $+5,73$ \\
Calidad de vida residencial & $+2,85$ \\
Arquitectura de marketing & $+2,71$ \\
Medio ambiente y sostenibilidad & $+2,69$ \\
Participación & $+2,40$ \\
Integración social & $+1,59$ \\
Nueva Economía & $+1,26$ \\
\hline
\end{tabular}

FUENTE ELABORACIÓN PROPIA.

El cuadro recoge las actuaciones que han tenido un mayor incremento en el nuevo modelo de ciudad. En su conjunto, tienen como denominador común:

a La búsqueda de calidad de vida urbana en todas sus dimensiones (medio ambiente, calidad de las áreas residenciales, integración social, parques) y se relacionan con el programa de ciudad habitable.

b La gobernabilidad o gobernanza (aumentan las actuaciones encaminadas a mejorar la participación de los ciudadanos en el gobierno de la ciudad), casi siempre centradas en el movimiento asociativo.

c Las actuaciones para la promoción de la nueva economía del conocimiento (parques científicos, centros de alta formación, centros de investigación aplicada al sistema urbano local) o, lo que es lo mismo, la ciudad del conocimiento.

d Por encima de todas, las estrategias de marketing urbano (imagen, marca y arquitectura), muy relacionadas con el fenómeno de arquitectura-escaparte.

2 Las actividades que han perdido peso en las estrategias urbanas postindustriales fueron las que se exponen en la siguiente tabla.

CUADRO 4 | Las actividades que han perdido prioridad

\begin{tabular}{lc}
\hline TIPO DE ACTUACIONES & DIFERENCIA B-A \\
\hline Turismo y ocio & $-5,52$ \\
Renovación morfológica & $-5,09$ \\
Atractivo empresarial & $-4,92$ \\
Infraestructuras & $-2,98$ \\
Eventos & $-0,50$ \\
Cultura & $-0,19$ \\
Cooperación capital privado & $-0,02$ \\
Actividades de I+D & $-0,02$ \\
\hline
\end{tabular}


1 Ocupan el primer lugar las relacionadas con el turismo y el ocio. En la transición postindustrial todas las ciudades, como hemos visto, aspiraron a diversificar su actividad productiva y avanzar en el modelo de la ciudad de servicios para incrementar el número de visitantes, lo que les llevó a hacer hincapié en actividades turísticas y de ocio. La realidad nos dice dos cosas: por un lado, que este objetivo no se consiguió en todos los casos, ni alcanzó la importancia económica que se esperaba y, por otro, que una vez creados los equipamientos no fue necesario continuar esta línea, bien porque la demanda ha sido inferior a las previsiones, o bien porque una vez alcanzado un cierto nivel de equipamiento el esfuerzo inversor se trasladó hacia el marketing estratégico urbano para promocionar dichos equipamientos.

2 También han perdido peso las variadas actuaciones de renovación morfológica (centros históricos, áreas centrales, espacios portuarios o fluviales), porque en la mayoría de los casos estos objetivos fueron los primeros que se acometieron al iniciar el cambio urbano, y sólo los introdujeron o mantuvieron las ciudades que no terminaron los proyectos de rehabilitación, o que no estuvieron adecuadamente conducidos. También hay que considerar el caso de las ciudades donde se retrasó su puesta en marcha por dar preferencia al equipamiento de los barrios periféricos, más necesitados de inversiones que el centro. Se trata, en todo caso, de actuaciones de continuidad.

3 Algo parecido ocurrió con las actuaciones orientadas a atraer nuevas actividades empresariales. La causa también es doble. De un lado, porque los equipamientos e infraestructuras ya estaban hechos en la primera fase y, de otro, porque fueron muy pocas las ciudades que lograron resultados notables en este ámbito de intervención, de cuyo aparente fracaso se deriva la pérdida de interés práctico. Pero, en la realidad, hay que tener en cuenta que se trata de objetivos que deben evaluarse a largo plazo, por lo cual, es difícil obtener resultados en el corto. Lo mismo ocurre con las actividades de I+D+I.

4 En otro orden de cosas, están aquellas actuaciones que respondían a déficits coyunturales y que en parte ya habían sido cubiertos en la fase de ajuste (infraestructuras, equipamientos culturales, cooperación privada), y que sólo se añaden en los proyectos urbanos de nueva implantación. Por otro lado, se produjo una redundancia de actuaciones como consecuencia de que todas las ciudades programaran los mismos objetivos (cultura, eventos, congresos, turismo, I+D, sedes de empresas, etc.), haciendo que la reiteración de los mismos en todos los lugares hiciera perder competitividad, dado el carácter selectivo del segmento de demanda de tales localizaciones. 


\section{Conclusiones derivadas}

De la comparación entre las actuaciones urbanas realizadas en la etapa de transición y las realizadas en la etapa postindustrial y global, se extraen las siguientes conclusiones:

a Existen dos tipos de actuaciones urbanas: imagen y marca $(+5,73)$ y arquitectura de marketing $(+2,71)$, que han experimentado el mayor crecimiento en la fase postindustrial en relación con la etapa de transición. Este tipo de actuaciones pertenecen al ámbito del marketing urbano.

b Otro grupo de actuaciones que ha experimentado un fuerte crecimiento en la segunda etapa son las relacionadas con la mejora de la habitabilidad, materializadas en: calidad de vida residencial $(+2,85)$ y medio ambiente y sostenibilidad $(+2,69)$.

c Las actuaciones encuadradas dentro del turismo y ocio $(-5,52)$ y la promoción de nuevas actividades económicas son las que han descendido más en peso específico en la segunda etapa, en relación con la primera. La causa más probable es que al ser objetivos que no dependen exclusivamente de la inversión pública, sino que requieren un tiempo de maduración mayor y obedecen a procesos de cambio muy complejos, pierden interés para los políticos y planificadores que trabajan a corto y medio plazo. De hecho, muy pocas ciudades de las examinadas han logrado recuperar el peso económico que antes habían tenido, a no ser en los casos con bases tecnológicas y de conocimiento de fuerte implantación en la ciudad.

d Las actuaciones de renovación morfológica $(-5,09)$ e infraestructuras $(-2,98)$ también experimentan un importante descenso en relación con la etapa de transición.

En suma, empíricamente se observa que las líneas de actuación de las ciudades han evolucionado desde los planteamientos y las herramientas de la Planificación Estratégica hacia otros más propios del ámbito del Marketing Urbano. Por lo tanto, el centro de atención se ha desplazado desde los aspectos materiales de las ciudades (morfología e infraestructuras) a la valorización de los aspectos inmateriales. En consecuencia, las conclusiones del análisis empírico coinciden con las observadas al efectuar el estudio de casos. Por eso, se puede concluir que se está produciendo una valorización de los activos inmateriales de la economía y la sociedad urbana, que deberá conducir a los planificadores a poner en marcha nuevas metodologías de participación y creatividad social, tal como al final de la primera parte habíamos planteado. IEURE 


\section{Referencias bibliográficas}

Álvarez, M. A. (2005). Patrimonio industrial y política cultural en el marketing de ciudades y territorios Ábaco. Revista de Cultura y Ciencias Sociales, 44-45, pp. 45-62.

Anguiano, R. (2005). Experiencias urbanas en México y Colombia. Ábaco. Revista de Cultura y Ciencias Sociales, 44-45, pp. 115-118.

Ashworth, G. J. (1993). Selling the City: Marketing Approaches in Public Sector Urban Planning. New York: John Wiley \& Sons.

Ashworth, G. J. \& Voogd, H. (1990). Selling the City: Marketing Approaches in Public Sector Urban Planing. London: Belhaven.

Benko, G. (2000). Estrategias de comunicación y marketing urbano. Eure. Revista Latinoamericana de Estudios Urbano Regionales, Vol. XXVI, 79, pp. 67-76.

Blume, L. (2006). Local economic policies as determinants of the local business climate: empirical results from a cross-section analysis among East German municipalities. Regional Studies, 40(4), pp. 321-333.

Bornemeyer C. y Decker, R. (2001). Key success factors in city marketing -some empirical evidence. Exploratory Data Analysis in Empirical Research Proceedings, pp. 443-453.

Bramwell, B. y Rawding, L. (1994). Tourism marketing organizations in industrial cities - organizations, objectives and urban governance. Tourism Management, 15(6), pp. 425-434.

Berg, L. van den; Braun, E. \& Otgaar, A. H. J. (2002). Sports and city marketing in European cities. Aldershot: Ashgate.

Broudehoux, A. M. (2007). Spectacular Beijing: the conspicuous construction of an Olympic metropolis. Journal of Urban Affairs, 29(4), pp. 383-399.

Castells, M. (1996). The Information Age: Economy, Society and Culture. Vol I. The Rise of the Network Society. Cambridge, MA; Oxford, UK: Blackwell.

Chacón, R. M. \& Silva, D. (2005). Las ciudades hacia el desarrollo sostenible. Ábaco. Revista de Cultura $y$ Ciencias Sociales, 44-45, pp. 71-78.

Cubillo, J. M. \& Sánchez, J. (2005). Imagen-Ciudad y calidad percibida de los servicios. Ábaco. Revista de Cultura y Ciencias Sociales, 44-45, pp. 79-87.

Dahles, H. (1998). Redefining Amsterdam as a tourist destination. Annals of Tourism Research, 25(1), pp. 55-69.

Domínguez, P. \& Bernard, A. (2005). Hacia una ciudad histórico-turística legible. El desafío de Puebla de los Ángeles (México). Ábaco. Revista de Cultura y Ciencias Sociales, 44-45, pp. 89-95.

D'Orsi, P. M. (2005). City marketing y administración de ciudades o city management. El rol especial del city marketing en la administración de ciudades o city management. Ábaco. Revista de Cultura y Ciencias Sociales, 44-45, pp. 97-104.

Elizagarate, V. (1996). Marketing de ciudades. Una necesidad para los procesos de revitalización. Revista de Dirección y Administración de Empresas, n 4, noviembre, pp. 63-71.

Elizagarate, V. (2003). Marketing de ciudades. Madrid: Ediciones Pirámide.

Elizagarate, V. (2008). Marketing de ciudades. Estrategias para el desarrollo de ciudades atractivas y competitivas en un mundo global. Madrid: Ediciones Pirámide.

Fajardo, S. (2005). Medellín, una nueva cara. Abaco. Revista de Cultura y Ciencias Sociales, 44-45, pp. 122-126. 
Fernández, G. (2004). La imagen de ciudad como recurso para la gestión estratégica del desarrollo urbano. En G. Fernández y G. Leva (Eds.). Lecturas de economía, gestión y ciudad. Buenos Aires: Universidad Nacional de Quilmes.

Fernández, G. (2006). El ciudadano como creativo de marca de ciudad. Diseño y gestión de marcas para el desarrollo urbano y la participación ciudadana. Ponencia presentada en las III Jornadas de Marketing de Ciudades, 26-28 abril, A Coruña, España.

Fernández, G. \& Friedmann, R. (2007). Gestión y liderazgos en el diseño e implementación de planes estratégicos de marketing de ciudades: un balance y propuestas a partir de la experiencia alemana. Ponencia presentada en el IX Coloquio Internacional de Geocritica, 28 de mayo-1 de junio, Porto Alegre, Brasil.

Fernández, G. \& Leva, G. (Eds.) (2004). Lecturas de economía, gestión y ciudad. Buenos Aires: Universidad Nacional de Quilmes.

Fernández, G. \& Paz, S. (2005a). Más allá del marketing de ciudades: hacia una política pública de diseño y gestión de los signos de identificación de ciudad. Scripta Nova, Revista electrónica de geografia y ciencias sociales, Vol. IX, núm. 194 (94). http://www.ub.es/geocrit/sn/sn-194-94.htm

Fernández, G. \& Paz, S. (2005b). Desarrollo urbano, cultura de marca y participación ciudadana. Ábaco. Revista de Cultura y Ciencias Sociales, 44-45, pp. 63-70.

Fernández Güell, J. M. (1997). Planificación estratégica de ciudades. Barcelona: Editorial Reverté.

Fernández Güell, J. M. (2006). Planificación Estratégica de Ciudades. Nuevos Instrumentos y procesos. Barcelona: Reverté.

Franz, P. (2000). Suburbanization and the clash of urban regimes -Developmental problems of East German cities in a free market environment. European Urban and Regional Studies, 7(2), pp. 135-146.

Friedmann, R. (2004). Urban Management by Complexity. Nuevas formas de gestión estratégica urbana y de participación ciudadana. En G. Fernández y G. Leva (Eds.). Lecturas de economía, gestión y ciudad. Buenos Aires: Universidad Nacional de Quilmes.

Friedmann, R. (2005). Marketing estratégico de ciudades. Ábaco. Revista de Cultura y Ciencias Sociales, 44-45, pp. 21-27.

Friedmann, R. \& Fernández, G. (2007). Gestión y liderazgo municipal en la ciudad posmoderna. Ponencia presentada en las IV Jornadas de Marketing de Ciudades, 28-30 marzo, A Coruña, España.

González, S. (2006). Scalar narratives in Bilbao: a cultural politics of scales approach to the study of urban policy. International Journal of Urban and Regional Research, 30, Dec. 2006, pp. 836-857.

Green, R. (2005). Pensar, dibujar, matar la ciudad: orden, planificación y competitividad en el urbanismo moderno. EURE. Revista Latinoamericana de Estudios Urbano Regionales, Vol. XXXI, núm. 94, pp. 77-95.

Hall, P. (1993). Forces shapping urban Europe. Urban Studies, 30, pp. 883-898.

Hall, P. (2001). Ciudad para la sociedad del siglo XXI. Valencia: Colegio Oficial de Arquitectos de la Comunidad Valenciana.

Iwata, N. \& del Río, V. (2004). The image of the waterfront in Rio de Janeiro urbanism and social representation of reality. Journal of Planning Education and Research, 24(2), pp. 171-183.

Kavaratzis, M. \& Ashworth, G. J. (2007). Partners in coffeeshops, canals and commerce: marketing de city of Amsterdam. Cities, 24(1), pp. 16-25. 
Leva, G. (2004). Globalización, competitividad internacional y ciudad. En G. Fernández y G. Leva (Eds.) Lecturas de economía, gestión y ciudad. Buenos Aires: Universidad Nacional de Quilmes.

Limburg, B. van. (1998a). The study of the effects of price on incoming overnight tourism in the Netherlands and Amsterdam -the case of USA tourists. Tourism Management, 19(1), pp. 87-91.

Limburg, B. van. (1998b). City Marketing: a multi-attribute approach. Tourism Management, 19(5), pp. 475-477.

Martínez, A. (2004). Planificación estratégica y la imagen de la ciudad. Ponencia presentada en el $I$ Congreso Citymarketing Elche'04, Elche, España.

Metaxas, T. (2003). The image of the city as good: the creation of a city's promotional package through a strategic framework analysis of city marketing procedure. Sustainable Planning and Development, 6, pp. 427-438.

Meyer K. (1979). Love the City-Marketing the american metrópolis. Saturday Review, 6, pp. 16.

Muñiz, N. (2008). Marketing de ciudades con fachadas marítimas y fluviales; urbanismo de excelencia y eventos internacionales. Ponencia presentada en el II Congreso Mundial de Marketing de Ciudades, Rosario, Argentina.

Muñiz, N. (2009). Marketing Estratégico de Ciudades. En J. L Munuera y A. I. Rodríguez (Eds.) Casos de marketing estratégico en las organizaciones. Madrid: Esic Editorial.

Muñiz, N. \& Cervantes, M. (2005). Las aportaciones del marketing a la gestión de ciudades. Ábaco. Revista de Cultura y Ciencias Sociales, 44-45, pp. 37-43.

Noissete, P. \& Vallerugo, F. (1996). Le Marketing des villes. París: Les Éditions d'Organisation.

Paddison, R. (1993). City Marketing, image-reconstuction and urban regeneration. Urban Studies, 30, pp. 339-350

Pancorbo, J. A. (2006). El marketing urbano como herramienta de apoyo en la gestión de los centros históricos. Ponencia presentada en las III Jornadas de Marketing de Ciudades, 26-28 abril, A Coruña, España.

Pancorbo de Sandoval, A. \& Hevia, A. (2004). La planificación estratégica territorial: una visión desde el marketing. En G. Fernández y G. Leva (Eds.) Lecturas de economía, gestión y ciudad. Buenos Aires: Universidad Nacional de Quilmes.

Paz, S. (2004). Marca territorial. Cómo construir y administrar la marca de ciudades y regiones. En G. Fernández y G. Leva (Eds.) Lecturas de economía, gestión y ciudad. Buenos Aires: Universidad Nacional de Quilmes.

Paz, S. (2006). Marca territorial. Valor para la política de promoción comercial. Buenos Aires: Politike Ediciones.

Precedo, A. (1996). Ciudad y desarrollo urbano. Madrid: Síntesis.

Precedo, A. (2004). Nuevas Realidades Territoriales para el siglo XXI desarrollo local, identidad territorial y ciudad difusa. Madrid: Síntesis.

Precedo, A.; Orosa, J. J. \& Míguez, A. (2007). El área metropolitana de A Coruña: una metrópoli Euratlántica. Estudio de posicionamiento, constitución y marketing. A Coruña: Diputación Provincial de A Coruña.

Precedo, A.; Orosa, J. J. \& Míguez, A. (2010). Marketing de Ciudades y producto ciudad: una propuesta metodológica. Urban Public Economics Review, en prensa. 
Reason, M. \& García, B. (2007). Approaches to the newspaper archive: content analysis and press coverage of Glasgow's Year of Culture. Media Culture \& Society, 29(2), pp. 304.

Rodell, M. J. (1993). City Marketing -The Bottom line. Third World Planning Review, 15, pp. 187-193. Sassen, S. (1991). The global city. New York, London, Tokyo: Princeton University Press.

Sassen, S. (2007). El reposicionamiento de las ciudades y regiones urbanas en una economía global: ampliando las opciones políticas y gobernanza. Eure. Revista Latinoamericana de Estudios Urbano Regionales, Vol. XXXIII, núm. 100, pp. 9-34.

Sánchez, F. \& Moura, R. (2005). Ciudades-modelo: estrategias convergentes para su difusión internacional. Eure. Revista Latinoamericana de Estudios Urbano Regionales, Vol. XXXI, núm. 93, pp. 21-34.

Seisdedos, H. (1999). Estudio de las políticas de comunicación de las entidades locales: una aproximación a la realidad del City Marketing en España. Ponencia presentada en el XI Encuentro de Profesores Universitarios de Marketing. Madrid: ESIC, pp. 381-384.

Seisdedos, H. (2007). Cómo gestionar las ciudades del siglo XXI. Del City Marketing al Urban Management. Madrid: Pearson Educación.

Thornley, A. (1998). Institutional change and London's urban policy agenda. Annals of Regional Science, 32(1), pp. 163-183.

Yao, F.; Sun, L.Y. \& Yao, P. (2005). Survey of urban marketing. Proceedings of 2005 International Conference on Public Administration, pp. 858-864. 